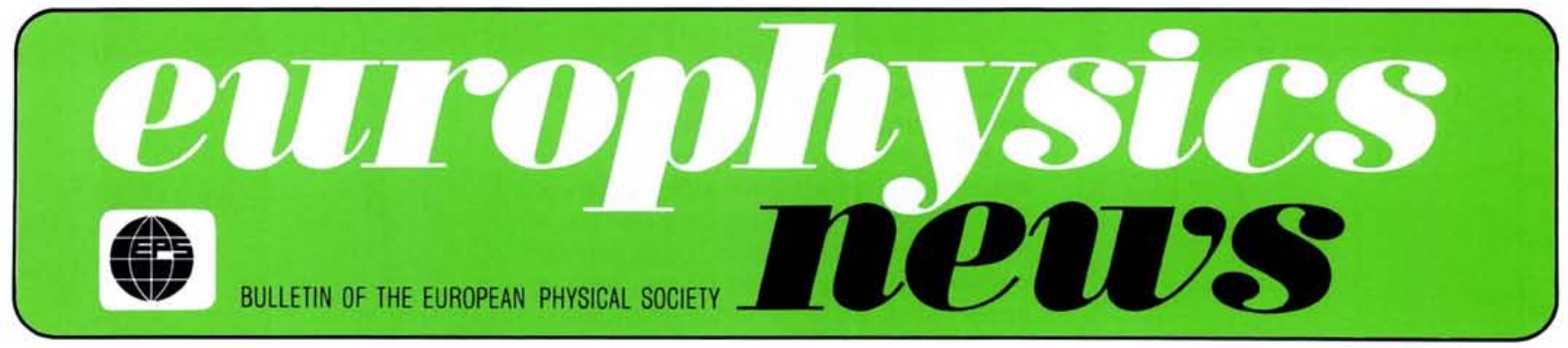

J.A.

\title{
Radiation of Channelled Positrons and Electrons; Possibilities of Application
}

\author{
R. Wedell, Berlin, DDR
}

(Humboldt University)

When a beam of charged particles traverses a crystal along closely packed atomic strings or planes, the particles undergo correlated collisions with the atoms (see Fig. 1) which leads to a constrained motion that is termed channelling. The interaction potential may be described as a sum of continuum atomic string or plane potentials in the two situations. The term is appropriate as a fast particle will undergo small angle scattering from a large number of atoms, the half-wavelength of the particle trajectory being of the order of 10-100 interatomic spacings (see Fig. 2). Neglecting multiple scattering and energy loss effects, the transverse energy $E_{\perp}$ in the plane perpendicular to the considered channel direction is conserved, i.e.

$$
E_{\perp}=\frac{\vec{p}_{\perp}^{2}}{2 m_{\mathrm{e}} \gamma}+V(\vec{\rho})=\text { const. }
$$

Fig. 2 - Motion of an axial or planar channelled particle close to an atomic string or plane.
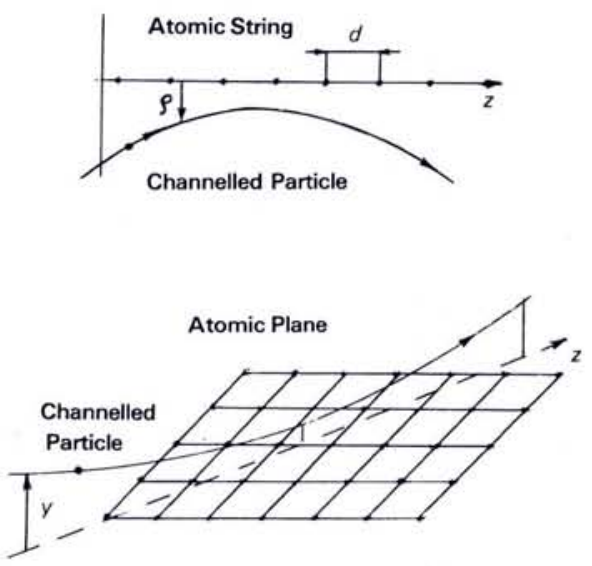

where $\overrightarrow{p_{\perp}}$ is the transverse momentum, $\gamma=$ $E / m_{e} c^{2}$ the Lorentz-factor, $E$ the total particle energy and $V(\vec{\rho})$ the continuum channel potential.

The basic theoretical description of channelled particle motion based on classical methods was given by Lindhard ${ }^{1}$ ) in 1965 and at the turn of the 1960's the channelling of positrons and electrons was observed. At relativistic energies, the particle behaviour can be described classically, whereas at lower energies, quantum effects become significant.

In 1976, M.A. Kumakhov ${ }^{2}$ ) predicted that channelled positrons or electrons of relativistic energies would radiate, and that as the electric field created by atomic strings or planes is of the order of $10^{10}-10^{11}$ $\mathrm{V} / \mathrm{cm}$, the radiation should be intense. While the characteristic frequencies ${ }^{2}$ ) of particle trajectory oscillations in channels is $10^{15}-10^{16} / \mathrm{s}$, the radiation frequencies, owing to the Doppler effect, are shifted to the $\mathrm{X}$ and $\gamma$-ray region.

\section{Theoretical Aspects}

Consider the case of the planar channelling of positrons. To a first approximation, the continuum potential in the channel may be taken as a harmonic $V(x)=V_{o} x^{2}$, where $x$ is the distance from the channel centre. The particle motion can then be expressed as:

$$
\begin{aligned}
& x(t)=x_{m} \cos \left(\bar{\omega} t+\delta_{0}\right) \\
& z(t)=v_{z} t
\end{aligned}
$$

where: $\bar{\omega}=\Omega_{0} \gamma^{-1 / 2} ; \Omega_{0}=\sqrt{ }\left(2 V_{o} / m_{e}\right)$, the oscillation frequency neglecting relativistic effects; $x_{m}$ the amplitude of the particle oscillation; $v_{z}$ the longitudinal velocity component.

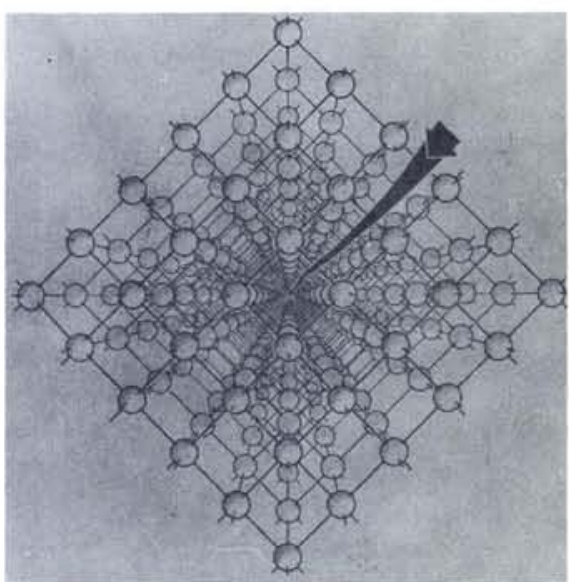

Fig. 1 - Illustration of the channelling effect.

As seen from (2, a and b), although the positrons have a relativistic velocity along the channel axis, they carry out nonrelativistic transverse oscillations. According to classical electrodynamics the total radiation intensity is given by

$$
I_{\mathrm{o}}=x_{m}^{2} \mathrm{e}^{2} \Omega_{0}^{4} \gamma^{2} / 3 c^{3} \cong \gamma^{2}
$$

This it will be seen increases proportionally with $E^{2}$. The oscillation frequency in the frame in which the longitudinal component of the particle velocity is zero, is given by

$$
\omega_{\mathrm{oo}}=\sqrt{ }\left[2 V_{\mathrm{o}}(\gamma)\right] / m_{\mathrm{e}}=\Omega_{\mathrm{o}} \gamma^{1 / 2}
$$

the factor $\gamma$ coming from the Lorentz transformation of the potential $V(x)$ in the laboratory frame, into the potential $V^{\prime}(x)=$ $\gamma V(x)$ in the frame which moves with the particle along the channel axis. The fre-

\section{Contents}

Radiation of Channelled Relativistic Positrons and Electrons

Computations in Plasma Physics

New Individual Members

Career Outlook for Physicists in Europe

Heinrich Barkhausen

Computational Physics Group

Plasma Physics Division

Official Announcements

1
4
7
8
10
11
11
12




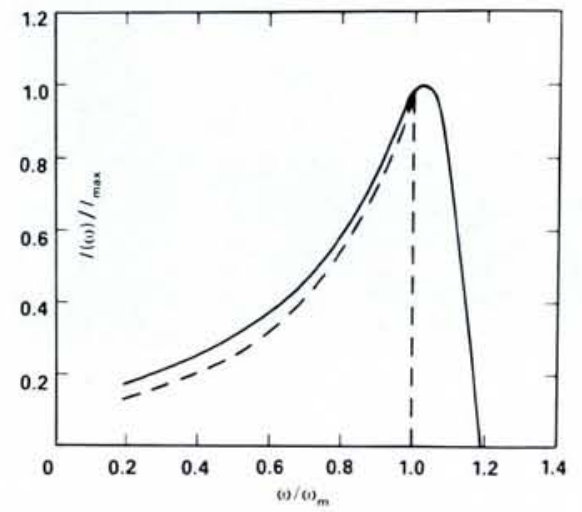

Fig. 3 - Radiation of channelled positrons in (110) Si, $E=1 \mathrm{GeV}, I_{\max }=6.1 \times 10^{18} \mathrm{Ws}$, $\omega_{m}=3.78 \times 10^{21} \mathrm{~s}^{-1}$; dashed curve harmonic potential, solid curve anharmonic potential (ref. 3).

quency of the emitted radiation at an angle $\theta$ taking into account the Doppler effect is then derived from:

$\omega(\theta)=\omega_{\mathrm{oo}} \sqrt{ }\left(1-v^{2} / c^{2}\right) /[1-(v / c) \cos \theta]$

which gives:

$\omega(\theta)=\left(\Omega_{0} \gamma^{-1 / 2}\right) /\left(1-\beta_{z} \cos \theta\right)(6)$ where $\beta_{z}=v_{z} / c$.

At $\theta=0$ we have the maximum frequency

$\omega_{m}=\left(1+\beta_{z}\right) \Omega_{0} \gamma^{3 / 2}=2 \Omega_{0} \gamma^{3 / 2}$

Fig. 3 gives the spectrum from $1 \mathrm{GeV}$ positrons channelled over the (110) plane in silicon, wherein the solid curve was obtained taking into account the anharmonicity of the potential. The sharp maximum that can be seen at $\omega=\omega_{m}$ means that the radiation is largely monochromatic.

In axial channelling, the particles carry out a two-dimensional transverse motion, as the channels are formed by atomic strings rather than by planes. They may be considered in two groups: those with relatively high transverse energies, which are scattered by separate atomic strings and may pass from one channel to another, radiating relatively hard quanta; those with small transverse energies which are the real channelled positrons, and which radiate photons of lower energies. The resulting spectrum is more spread out than with planar channelling. Furthermore, several maxima may be observed owing to the presence of several groups of particles with different characteristic frequencies.

The peculiarities of electron channelling and its radiation arise from the attractive interaction potential between the electrons and atomic strings or planes; electrons cross and recross the atomic planes rather than going between. Near to the planes, the interaction potential is to a high degree anharmonic and this results in the appearance of various maxima in the radiation spectrum. Furthermore as the potential gradients for electron channelling are larger than for positron channelling, the radiation intensity is higher.
In axial electron channelling, the transverse motion is elliptical and the particles spiral around the atomic strings. Such a motion is called a "rosette motion" and is described by an effective string potential $W(r)=L^{2} /\left(2 m_{\mathrm{e}} \gamma r^{2}\right)+U(r)$, which has a local minimum. The value $L$ (the angular momentum), is governed by the detailed position and angle at which the particle enters the string. A bound motion is only possible over the interval given by

$$
r_{\mathrm{cr}} \leq r \leq D / 2
$$

where $r_{c r}$ is the minimum approach of an electron to the string in channelling and $D$ is the distance between atomic strings. Consequently only a part of the electron beam is captured in the channelling regime, the number being strongly influenced by the beam divergence. Nevertheless, under optimal conditions, more than $50 \%$ of the electron beam may be captured. Because the electrons carry out a rosette motion around the atomic strings, the radiation is axially symmetric provided the targets have sufficient depth. The intensity of the radiation with planar channelling exceeds that of the positron radiation by an order of magnitude, and the radiation is harder for the same energy.

Despite the differences described above between the radiation from relativistic channelled positrons and electrons, the main characteristics of the dipolar Kumakhov radiation are the same when $E<\left(m_{\theta} c^{2}\right)^{2} / U_{0}$ and $U_{0}$ is the height of the potential barrier between channels $\left(E_{\mathrm{cr}} \sim\right.$ $10 \mathrm{GeV}$ for positrons and $1 \mathrm{GeV}$ for electrons). These characteristics can be summarized as follows:

(i) Total intensity $I_{0} \propto E^{2}$ (provided classical electrodynamics is applicable);

(ii) Maximum frequency $\omega_{m} \propto E^{3 / 2}$;

(iii) Emitted frequency at angle $\theta$ is given by (6).

In a quantum-mechanical treatment ${ }^{3}$ ), the transverse motion of channelled relativistic positrons or electrons can be described by a nonrelativistic Schrödinger equation with a relativistic particle mass:

$$
\begin{aligned}
{\left[-\frac{\hbar^{2}}{2 m_{\mathrm{e}} \gamma} \Delta+\right.} & \left.V\left(\overrightarrow{r_{\perp}}\right)\right] \phi_{n}\left(\overrightarrow{r_{\perp}}\right) \\
& =E_{\perp n} \phi_{n}\left(\overrightarrow{r_{\perp}}\right)
\end{aligned}
$$

The Kumakhov radiation is then considered to be due to transitions between the energy levels $E_{\mathrm{Ln}}$ which are formed in the field of atomic strings or planes. Quantum mechanical calculations of radiation characteristics agree with classical results in the quasiclassical limit.

\section{Experimental Results}

In November 1978, a Soviet-American experiment ${ }^{4}$ ) was carried out in Stanford (USA) with positron beams of energy 4,6 , 10 and $14 \mathrm{GeV}$ using as targets, diamond crystals with a thickness of 80 and $600 \mu \mathrm{m}$. Fig. 4 shows the spectral density per positron unit path obtained when $6 \mathrm{GeV}$ positrons were beamed into the $80 \mu \mathrm{m}$ target in

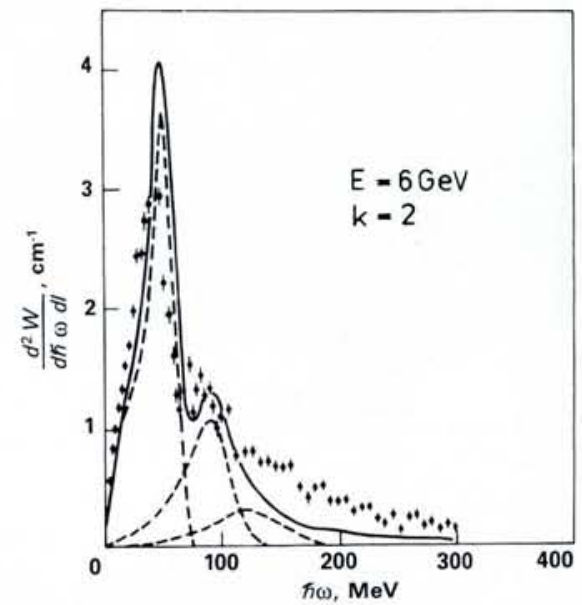

Fig. 4 - Spectral density of radiation energy from one positron per unit path in the planar channel (110): $E=6 \mathrm{GeV}$. Solid curves: calculated spectra; dashed curves: contributions from different radiation harmonics (ref. 4).

the (110) planar channel. The experimental results are compared with a theoretical curve obtained by dividing the calculated spectra by a parameter, in order to take account of the non-dipole character of the radiation which leads to a decrease in intensity. For the maximum frequency $\omega_{m}$, agreement between the general theory (with allowance for the non-dipole character) and experiment is good. Moreover the predicted dependence of the maximum frequency on the incident energy $\left(\omega_{m} \propto E^{3 / 2}\right)$ is followed up to $10 \mathrm{GeV}$ although at 14 $\mathrm{GeV}$, deviations become pronounced.

Alguard et al. ${ }^{5}$ ) have observed radiation from $56 \mathrm{MeV}$ positrons channelled between the three major planes and along the $<110\rangle$ axis in an $18 \mu \mathrm{m}$ thick silicon crystal. The planar channelling spectra showed the typical peak of Fig. 3 whereas the axial spectrum had a broad maximum in the vicinity of $100 \mathrm{keV}$, which was also in accordance with expectations. The energy dependence of the maximum frequency $\omega_{m}$ $\propto E^{3 / 2}$ was also observed.

Fig. 5 - Ratio of the $\gamma$-quanta yield in channelling to the random yield for electrons of energy $4.7 \mathrm{GeV}$. Dots: axis <100>; crosses: plane (110); curves, theory; (ref. 6).

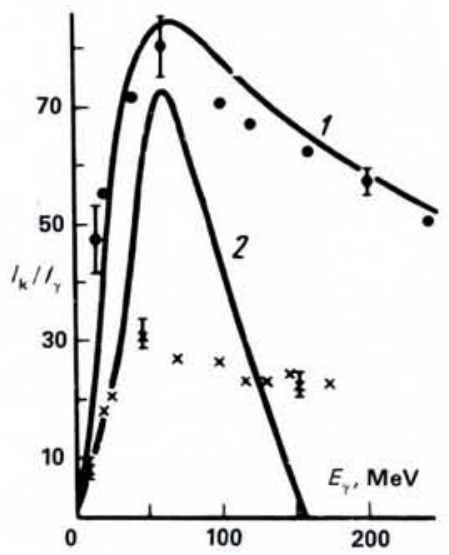




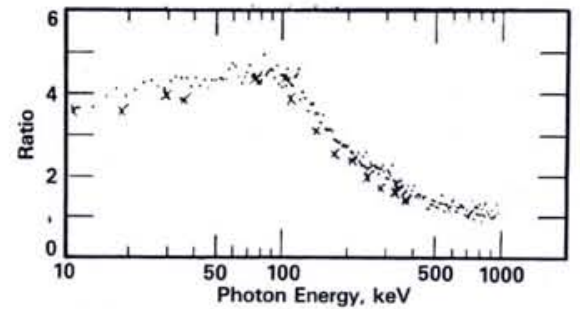

Fig. 6 - Ratio of the normalized axial electron channelling spectra to the corresponding random values in $\langle 110\rangle$ in Si. Dots: $E=28 \mathrm{MeV}$, crosses; $E=56 \mathrm{MeV}$ after reduction; (ref. 7).

The first experiments with electrons were carried out by Aganyants et al. ${ }^{6}$ ) in 1977 at the Erevan synchrotron with a diamond crystal as target. Beam energy was 4.7 $\mathrm{GeV}$, incident on the axial channel $<110\rangle$ and the planar channel (110). In Fig. 5 the observed spectra are compared with the calculated spectra, taking into account the non-dipole character. In the axial case, agreement is good, whereas in the planar case the deviations are pronounced.

A more detailed investigation was performed for 56 and $28 \mathrm{MeV}$ electrons channelled along the planes (100), (110) and (111) and the axis $\langle 110\rangle$ of an $18 \mu \mathrm{m}$ thick silicon crystal by Swent et al. ${ }^{7}$ ). Fig. 6 shows the ratio of the normalized axial channelling spectrum to the corresponding random spectrum for $28 \mathrm{MeV}$ electrons in $\langle 110\rangle$, and the reduced spectrum for the same channel at $E=56 \mathrm{MeV}$, obtained by dividing the intensity by a factor 2 and the photon energy by a factor $2^{3 / 2}$. Both spectra are in good agreement, which confirms the validity of the law $\omega_{m} \propto \gamma^{3 / 2}$.

With planar channelling, a number of spectral peaks were observed, but the correspondence between the calculated maxi$\mathrm{ma}$ and the experimental data was still satisfactory. The multiplicity of peaks can be explained by the high anharmonicity of the interaction potential.

More recently, radiation from 1.5 - 4 $\mathrm{MeV}$ electrons channelled along the $<111>$ direction in silicon has been observed $\left.{ }^{8}\right)$, confirming the theory that spectral maxima originate in transitions between bound states in the axial transverse potential (see Fig. 7), and that at low particle energies quantum mechanical considerations are important.

\section{Possible Applications}

Channelling radiation shows a high spectral density in a comparatively narrow frequency band and has a high degree of polarization. At the present time there are no powerful $\gamma$-ray sources in the energy range 0.1 to $100 \mathrm{MeV}$ as the intensity of synchrotron radiation decreases exponentially in this region, and bremsstrahlung has too broad a spectrum and angular distribution owing to the multiple scattering in a thick target. Channelling radiation has a spectral density similar to bremsstrahlung, so it should be possible to generate powerful

\title{
NOR DTt nordisk institut for teoretisk atomfysik Danmark · Finland · Island · Norge · Sverige
}

\section{POSTDOCTORAL POSITION}

\author{
NORDITA, Copenhagen
}

A postdoctoral position is available in the condensed matter theory group at NORDITA for the academic year 1982-83, with reappointment possible for the following year. The successful applicant will be expected to do research in one or more of the several areas in which NORDITA's staff is active and to interact with the faculty and visitors on various topics of mutual interest. At present, the faculty consists of $\mathrm{J}$. Hertz, A. Luther, J. Nørskov and C.J. Pethick, and current research topics include statistical mechanics of disordered and low-dimensional systems, quantum fluids, and surface electronic structure. Applicants should send a curriculum vitae and publication list and have two letters of recommendation sent to any one of the faculty members named above at:

\section{NORDITA \\ Blegdamsvej 17 \\ DK-2100 Copenhagen $\varnothing$}

All information must be received by 10 February 1982 .

fluxes of narrow frequency and angular spread.

At particle energies of several $\mathrm{MeV}$, the spectral density begins to exceed that of bremsstrahlung and intense beams of $X$-rays are emitted. There should be applications therefore in X-ray lithography. Already the use of synchrotron radiation has led to an improvement in resolving power and hence to a decrease in size of integrated circuits. The required frequency range can be reached with considerably smaller particle energies using channelling radiation. Furthermore, the exposure time could be reduced down to a few minutes.

An interesting possibility is to induce laser action through transitions between energy levels in the transverse potential well, the relative populations of the corresponding quantum states being influenc-

Fig. 7 - Comparison of measured line energies with calculations. Dashed curves: transitions from free states; inset: transitions between levels in <111> axial potential; vertical scale gives binding energy in rest frame (ref. 8 ).

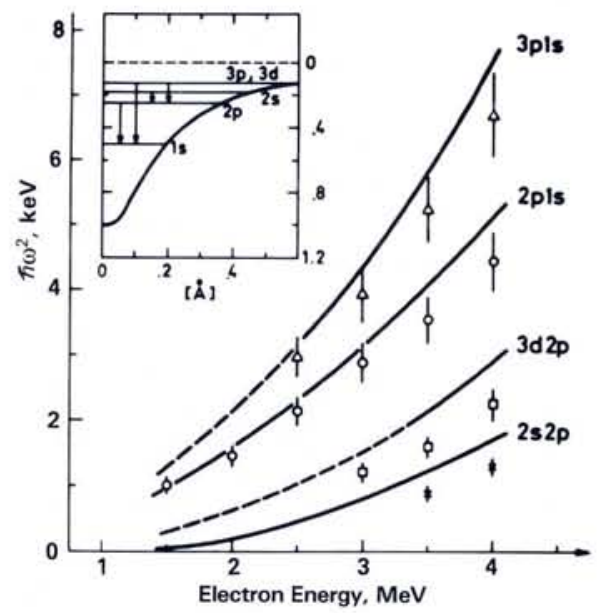

ed by the incident angle of the particle beam. Higher lying states can be made to have larger populations (inversion) giving rise to the laser emission of photons ${ }^{9}$ ). Further applications can be found in Ref. 10 .

As seen from the examples given, the unique properties of channelling radiation suggest a number of practical applications, and it will be of great interest to study experimentally, channelling over a wide energy region ( $1 \mathrm{MeV}<E<100 \mathrm{GeV}$ ) in a variety of target materials, thicknesses and channel direction.

\section{REFERENCES}

1. Lindhard J., Kong. Danske. Vid. Selsk., Mat. Fys. Medd., 34 (1965) 14.

2. Kumakhov M.A., Phys. Lett. A, 57 (1976) 17 Phys. Stat. Sol. (b), 84 (1977) 41.

3. Kumakhov M.A. and Wedell R., Phys. Lett. A, 59 (1976) 403; Phys. Stat. Sol. (b), 84 (1977) 581.

4. Miroshnishenko I.I., Murray J., Avakian R.O. and Fieguth T., Zh. Eksper. Teor. Fiz., Pisma, 29 (1979) 786 ;

see also Bazylev V.A., Beloshitsky V.V., Glebov V.I., Zhevago N.K., Kumakhov M.A. and Trikalinos Chr., Zh. Eksper. Teor. Fiz., 80 (1981) 608. 5. Alguard M.J., Swent R.L., Pantell R.H., Berman B.L., Bloom S.D. and Datz S., Phys. Rev. Lett., 42 (1979) 1148

6. Aganyants A.O., Vartanov Yu.A., Vartapetyan G.A., Kumakhov M.A., Trikalinos Chr. and Yarolov V.Ya., Zh. Eksper. Teor. Fiz. Pisma, 29 (1979) 554 (see also the references given in this paper)

7. Swent R.L., Pantell R.H., Alguard M.J., Berman B.L., Bloom S.D. and Datz S., Phys. Rev. Lett., 43 (1979) 1723.

8. Andersen J.U. and Laegsgaard E., Phys. Rev. Lett., 44 (1980) 1079.

9. Temmer G., Chinese Journal of Nuclear Phy. sics, (1980).

10. Wedell R., Phys. Stat. Sol. (b), 99 (1980) 11. 